\title{
Corrigendum: Graphene-enabled electrically switchable radar-absorbing surfaces
}

Osman Balci, Emre O. Polat, Nurbek Kakenov \& Coskun Kocabas

Nature Communications 6:6628 doi: 10.1038/ncomms7628 (2015); Published 20 Mar 2015; Updated 16 Nov 2015

The financial support for this article was not fully acknowledged. The acknowledgements should have included the following: O.B. and N.K. acknowledges TUBITAK-BIDEB for the 2211 and 2215 scholarship program. 